\title{
FOLFIRI and regorafenib combination therapy with dose escalation of irinotecan as fourth-line treatment for patients with metastatic colon cancer according to UGTIAI genotyping
}

\author{
This article was published in the following Dove Press journal: \\ OncoTargets and Therapy \\ 21 November 2014 \\ Number of times this article has been viewed
}

\author{
Chien-Yu Lu' ${ }^{1,2}$ \\ Yung-Sung Yeh ${ }^{3-5}$ \\ Ching-Wen Huang ${ }^{5,6}$ \\ Cheng-Jen $\mathrm{Ma}^{4,5}$ \\ Fang-Jung $\mathrm{Yu}^{1,2}$ \\ Jaw-Yuan Wang ${ }^{4-10}$ \\ 'Division of Gastroenterology, \\ Department of Internal Medicine, \\ ${ }^{2}$ Department of Internal Medicine, \\ Faculty of Medicine, College of \\ Medicine, ${ }^{3}$ Department of Emergency \\ Medicine, Kaohsiung Municipal Hsiao- \\ Kang Hospital, ${ }^{4} \mathrm{Graduate}$ Institute of \\ Clinical Medicine, College of Medicine, \\ ${ }^{5}$ Division of Gastroenterology and \\ General Surgery, Department of \\ Surgery, Kaohsiung Medical University \\ Hospital, ${ }^{6}$ Graduate Institute of \\ Medicine, College of Medicine, \\ ${ }^{7}$ Cancer Center, Kaohsiung Medical \\ University Hospital, ${ }^{8}$ Department \\ of Genomic Medicine, ${ }^{9}$ Department \\ of Surgery, College of Medicine, \\ Kaohsiung Medical University, \\ Kaohsiung, Taiwan; ${ }^{10} \mathrm{Center}$ for \\ Biomarkers and Biotech Drugs, \\ Kaohsiung Medical University, \\ Kaohsiung, Taiwan
}

Correspondence: Jaw-Yuan Wang Division of Gastroenterology and General Surgery, Department of Surgery, Kaohsiung Medical University Hospital, Kaohsiung Medical University, 100 Tzyou-You Ist Road, Kaohsiung City 807, Taiwan

Tel +88673122805

Fax +88673 II 4679

Email jawyuanwang@gmail.com

\begin{abstract}
Here we report a case of metastatic colon cancer treated with 5-fluorouracil, leucovorin, and escalated doses of irinotecan (FOLFIRI) combined with regorafenib in the fourth-line setting after uridine diphosphate glucuronosyltransferase (UGT)1A1 genotyping analysis. A 66-year-old male was initially diagnosed with Union Internationale Contre le Cancer stage III descending colon cancer and underwent curative surgery. He received postoperative adjuvant chemotherapy; however, liver metastasis developed and a partial hepatectomy was performed thereafter. Unfortunately, pulmonary metastases and recurrent liver tumors were found despite a series of systemic treatments with multiple combinations of cytotoxic and biologic agents. Recently, a novel multikinase inhibitor, regorafenib, was approved for the treatment of metastatic colorectal cancer refractory to other therapeutic modalities. As further treatment, we combined regorafenib with FOLFIRI, which included dose escalations of irinotecan, after UGT1A1 genotyping analysis. The therapeutic results were promising, with the improvement in liver and pulmonary metastases being classified as stable disease and partial response, respectively. Moreover, the progression-free survival was over 6 months. FOLFIRI, with dose escalation of irinotecan according to UGT1A1 genotyping plus regorafenib appears to be a promising salvage therapy for patients with refractory metastatic colorectal cancer.

Keywords: UGT1A1, irinotecan, dose escalation, metastatic colon cancer, regorafenib
\end{abstract}

\section{Introduction}

Approximately $20 \%-25 \%$ of patients with colorectal cancer (CRC) present with metastatic disease at the time of diagnosis, and up to $40 \%$ of those with initial limited CRC will develop metastases during the course of treatment, resulting in $50 \%-60 \%$ of CRC patients expected to develop metastases subsequently. ${ }^{1,2}$ Most of these patients with metastatic CRC will require intensive or palliative systemic therapy. The current first-line treatment for metastatic CRC includes the combination of 5-fluorouracil/ leucovorin and either oxaliplatin (FOLFOX) or irinotecan (FOLFIRI), which achieves a median survival of up to 20 months, but the 5-year survival rate does not exceed $10 \%{ }^{2,3}$ Addition of biologic agents, such as bevacizumab or cetuximab, can improve the survival outcomes when compared with chemotherapy alone. ${ }^{4,5}$ However, there is an unmet therapeutic need for patients with metastatic CRC who progress despite treatment with standard first-line, second-line, and third-line therapies. Here we report a case of refractory metastatic CRC with disease that progressed to liver and lung metastases after liver metastasectomy, third-line chemotherapy, and treatment with 
two biologic agents. This patient was started on a novel multikinase inhibitor, regorafenib, which was approved by the US Food and Drug Administration in 2012 and also by the Department of Health in Taiwan, in 2013 to treat refractory metastatic CRC.

\section{Case report}

A 66-year-old male visited our outpatient clinic with the complaints of post-prandial abdominal fullness, change in bowel habit, and small caliber stool for several months. He had no particular medical diagnosis, but family history revealed that his father had had colon cancer. Physical examination did not show specific findings. Colonoscopy revealed a circumferential tumor with a narrow lumen at the descending colon, and the pathologic biopsy showed adenocarcinoma. Abdominal computed tomography showed focal wall thickening with adjacent fat plane infiltration in the distal descending colon. The patient was clinically diagnosed with descending colon cancer, Union Internationale Contre le Cancer stage IIIB, based on the images and pathologic report. The carcinoembryonic antigen level was $36.6 \mathrm{ng} / \mathrm{mL}$ at diagnosis. Curative surgery was performed, and a post-resected specimen showed metastatic lymphadenopathy under histologic examination. The final pathologic stage was Union Internationale Contre le Cancer stage IIIB, T3N1aM0. Postoperative adjuvant chemotherapy with a regimen of capecitabine plus oxaliplatin (XELOX regimen) was prescribed for the patient.

After adjuvant chemotherapy, hepatic metastases were continuously suspected after a series of radiologic imaging examinations and elevated levels of carcinoembryonic antigen found during follow-up. Systemic chemotherapy with biweekly FOLFIRI (180 mg/m² dose of irinotecan) plus cetuximab $500 \mathrm{mg} / \mathrm{m}^{2}$ (the patients was identified as $K R A S$ wild-type according to the tumor specimen) was administered to the patient in the following therapeutic period. During this time, liver metastasectomy was also performed after a partial response was achieved with FOLFIRI plus cetuximab. However, lung metastases developed 6 months after the liver metastasectomy was performed. Subsequently, second-line systemic chemotherapy for this patient was shifted to biweekly FOLFIRI ( $180 \mathrm{mg} / \mathrm{m}^{2}$ dose of irinotecan) plus bevacizumab $5 \mathrm{mg} / \mathrm{m}^{2}$ for 12 cycles. Unfortunately, recurrent liver metastatic tumors and progressively elevated carcinoembryonic antigen levels (up to $708.09 \mathrm{ng} / \mathrm{mL}$ ) were noted after 6 months of FOLFIRI plus bevacizumab therapy. Despite third-line chemotherapy with FOLFOX4 ( $85 \mathrm{mg} / \mathrm{m}^{2}$ dose of oxaliplatin) plus bevacizumab $5 \mathrm{mg} / \mathrm{m}^{2}$, progressive disease was observed in this patient after four cycles of treatment.

Finally, this patient received FOLFIRI plus a newly approved biologic agent, regorafenib, in the fourth-line setting and had a partial response of distant metastases. The hepatic metastases were stable, but the pulmonary metastases improved (Figure 1A and B). In addition, the carcinoembryonic antigen level dropped markedly from $708.09 \mathrm{ng} / \mathrm{mL}$ to $97.42 \mathrm{ng} / \mathrm{mL}$ after addition of regorafenib to FOLFIRI. Regorafenib was administered to the patient at a dose of $160 \mathrm{mg}$ daily for the first 21 days of each 28-day cycle.

In regard to the escalating dose of irinotecan in the FOLFIRI regimen, genomic DNA extracted from the patient's peripheral blood was subjected to polymerase chain reaction sequencing of the promoter region of the uridine diphosphate glucuronosyltransferase (UGT)1A1 gene. The (TA)n repeat of this patient's gene showed $6 / 6$ repeats. In light of our previous UGT1A1 genotyping studies, ${ }^{6,7}$ the patient was treated with an initial $180 \mathrm{mg} / \mathrm{m}^{2}$ dose of irinotecan, administered over 2 hours on day 1 followed by leucovorin $\left(200 \mathrm{mg} / \mathrm{m}^{2}\right.$ as an intravenous infusion over 2 hours) and 5-fluorouracil (400 $\mathrm{mg} / \mathrm{m}^{2}$ as an intravenous bolus infusion followed by a 2,400 $\mathrm{mg} / \mathrm{m}^{2}$ intravenous infusion over a 46 -hour period), repeated every 2 weeks. The dose of irinotecan was gradually escalated up to $290 \mathrm{mg} / \mathrm{m}^{2}\left(180,210,240,260,290 \mathrm{mg} / \mathrm{m}^{2}\right)$ with a 20-30 mg/m² increment every two cycles of treatment, with
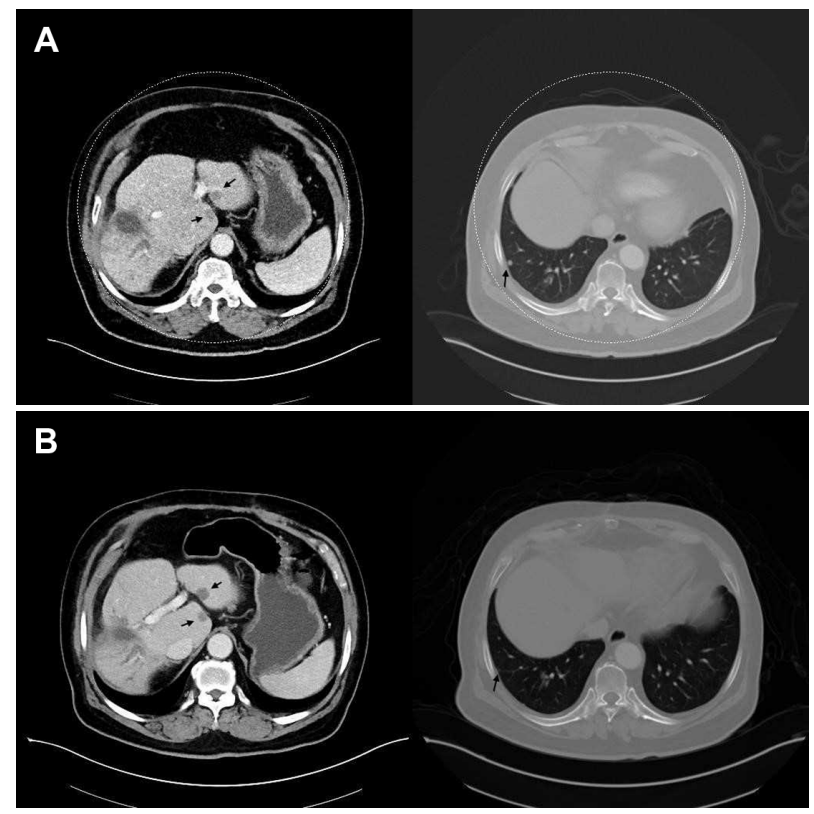

Figure I Abdominal computed tomography showing stable hepatic metastases (black arrow) but improved pulmonary metastases (black arrow) in our patient before $(\mathbf{A})$ and after $(\mathbf{B})$ treatment with FOLFIRI with irinotecan dose escalation plus regorafenib.

Abbreviation: FOLFIRI, irinotecan. 
acceptable adverse events for this patient. The therapeutic results were promising, with improvement in liver and pulmonary metastases being defined as stable disease and partial response, respectively; progression-free survival was longer than 6 months. The adverse events of FOLFIRI plus regorafenib were grade 2 hand-foot skin reaction and grade 1 fatigue.

\section{Discussion}

Irinotecan is converted to its active form, 7-ethyl-10hydroxycamptothecin (SN-38), in vivo, and exerts its cytotoxicity on dividing cancer cells. SN-38 is then further metabolized into an inactive metabolite, SN-38G, by the enzyme UDP-glucuronosyltransferase (UGT) in the liver. ${ }^{8}$ Glucuronidation of SN-38 to SN-38G is a decisive step in the metabolism and detoxification of irinotecan. However, genetic polymorphisms of the UGT1A1 gene can alter the glucuronidation of SN-38 and as such have a major influence on the pharmacokinetics and toxicity of irinotecan. ${ }^{9}$ The number of TATA box sequences in the UGT1A1 promoter alters the $U G T 1 A 1$ gene activity, ${ }^{10}$ with $6 \mathrm{TA}$ repeats representing the most common allele of the UGTIA1 gene (UGT1A1*1, wild-type), and 7TA repeats representing a variant allele (UGT1A1*28, mutant). The recommended dose of irinotecan in the FOLFIRI regimen is $180 \mathrm{mg} / \mathrm{m}^{2}$ every 2 weeks. However, this dose is insufficient for patients with the $U G T 1 A 1^{*} 1$ allele and contributes to a poorer clinical outcome. ${ }^{11,12}$ Our previous study demonstrated that patients receiving escalated doses of irinotecan achieved a better clinical response and outcome without significant adverse events. ${ }^{7}$ Furthermore, the role of irinotecan dose escalation is relatively more important in Asian races because they inherit the UGTIA1*28 allele less often than Caucasians. ${ }^{12,13}$

Regorafenib, a novel multikinase inhibitor targeting angiogenic, stromal, and oncogenic receptor tyrosine kinases, was developed and approved by the US Food and Drug Administration in September 2012 to treat patients with metastatic CRC who had failed to respond to FOLFOX, FOLFIRI, monoclonal anti-vascular endothelial growth factor, and anti-epidermal growth factor receptor pathway therapies. Regorafenib inhibits both intracellular and membrane-bound receptor tyrosine kinases, which are integral to angiogenesis, oncogenesis, and tumor growth/proliferation signaling. ${ }^{14}$ The Phase III Regorafenib Monotherapy for Previously Treated Metastatic Colorectal Cancer trial enrolled heavily pretreated patients with metastatic CRC like our reported case. A significant difference in the disease control rate, progression-free survival time, and an overall survival benefit for patients with metastatic CRC treated with regorafenib was demonstrated (41\% versus $15 \%, P<0.001 ; 1.9$ versus 1.7 months, $P<0.001$; 6.4 versus 5.0 months; $P=0.0052$, respectively).${ }^{15}$ The most commonly observed $\geq$ grade 3 adverse events include handfoot skin reaction, fatigue, hypertension, rash, and diarrhea. ${ }^{16}$ Two of the common side effects, ie, hand-foot skin reaction and fatigue, were also observed in our patient. Moreover, the active metabolite of irinotecan, $\mathrm{SN}-38$, is predominantly converted to an inactive metabolite, $\mathrm{SN}-38 \mathrm{G}$, by glucuronidation. As regorafenib is a strong inhibitor of glucuronosyltransferase in the liver, there is a potential role for pharmacokinetic interaction between irinotecan and regorafenib. ${ }^{17}$ Therefore, regorafenib might have a synergic effect with irinotecan and may enhance its cytotoxic capabilities. Using the combination of FOLFIRI (with irinotecan dose escalation) plus regorafenib, dual advantages can be anticipated, one being the synergic effect of regorafenib with irinotecan, and the other being the dose escalation of irinotecan by UGTIAl genotyping to achieve the most beneficial therapeutic outcome. Our patient with refractory metastatic CRC still had a partial response and progression-free survival for 6 months and longer with the combination of regorafenib and FOLFIRI (with escalated doses of irinotecan).

In conclusion, FOLFIRI (with irinotecan dose escalation) plus regorafenib has shown promising results in this patient with advanced and treatment-refractory disease after thirdline of treatment. However, a large sample size study is needed to highlight the potential advantage of combining FOLFIRI and regorafenib.

\section{Acknowledgments}

This work was supported by the Excellence for Cancer Research Center Grant through funding by the Ministry of Health and Welfare, Executive Yuan, Taiwan, Republic of China (MOHW103-TD-B-111-05), the Biosignature in Colorectal Cancers, Academia Sinica, Taiwan, the National Science Council, Republic of China (NSC 99-2320-B-037014-MY3), the Center for Biomarkers and Biotech Drugs, Kaohsiung Medical University (KMU-TP103C00, KMUTP103C03, KMU-TP103C07), and Kaohsiung Medical University Hospital (KMUH98-8G64, KMUH102-2R18).

\section{Disclosure}

The authors report no conflicts of interest in this work.

\section{References}

1. Arnold D, Stein A. New developments in the second-line treatment of metastatic colorectal cancer: potential place in therapy. Drugs. 2013;73(9):883-891. 
2. Van Cutsem E, Nordlinger B, Cervantes A. Advanced colorectal cancer: ESMO clinical practice guidelines for treatment. Ann Oncol. 2010; 21 Suppl 5:v93-v97.

3. Sanoff HK, Sargent DJ, Campbell ME, et al. Five-year data and prognostic factor analysis of oxaliplatin and irinotecan combinations for advanced colorectal cancer: N9741. J Clin Oncol. 2008;26(35): 5721-5727

4. Ocvirk J, Brodowicz T, Wrba F, et al. Cetuximab plus FOLFOX6 or FOLFIRI in metastatic colorectal cancer: CECOG trial. World $J$ Gastroenterol. 2010;16(25):3133-3143.

5. Hurwitz H, Fehrenbacher L, Novotny W, et al. Bevacizumab plus irinotecan, fluorouracil, and leucovorin for metastatic colorectal cancer. N Engl J Med. 2004;350(23):2335-2342.

6. Yeh YS, Huang ML, Chang SF, Chen CF, Hu HM, Wang JY. FOLFIRI combined with bevacizumab as first-line treatment for metastatic colorectal cancer patients with hyperbilirubinemia after UGT1A1 Genotyping. Med Princ Pract. 2014;23(5):478-481.

7. Lu CY, Huang CW, Hu HM, et al. Prognostic advantage of irinotecan dose escalation according to uridine diphosphate glucuronosyltransferase 1A1 (UGT1A1) genotyping in patients with metastatic colorectal cancer treated with bevacizumab combined with 5-fluorouracil/leucovorin with irinotecan in a first-line setting. Transl Res. 2014;164(2): 169-176.

8. Mathijssen RH, van Alphen RJ, Verweij J, et al. Clinical pharmacokinetics and metabolism of irinotecan (CPT-11). Clin Cancer Res. 2001;7(8): 2182-2194.

9. Iyer L, King CD, Whitington PF, et al. Genetic predisposition to the metabolism of irinotecan (CPT-11). Role of uridine diphosphate glucuronosyltransferase isoform 1A1 in the glucuronidation of its active metabolite (SN-38) in human liver microsomes. J Clin Invest. 1998;101(4):847-854.
10. Monaghan G, Ryan M, Seddon R, Hume R, Burchell B. Genetic variation in bilirubin UPD-glucuronosyltransferase gene promoter and Gilbert's syndrome. Lancet. 1996;347(9001):578-581.

11. Marcuello E, Paez D, Pare L, et al. A genotype-directed phase I-IV dose-finding study of irinotecan in combination with fluorouracil/ leucovorin as first-line treatment in advanced colorectal cancer. $\mathrm{Br} J$ Cancer. 2011;105(1):53-57.

12. Hebbar M, Ychou M, Ducreux M. Current place of high-dose irinotecan chemotherapy in patients with metastatic colorectal cancer. J Cancer Res Clin Oncol. 2009;135(6):749-752.

13. Tsai H-L, Chen C-F, Lu C-Y, et al. Significant correlation between polymorphisms of UGT1A1 gene and low irinotecan toxicity in colorectal cancer patients with FOLFIRI. Open Colorectal Cancer Journal. 2009;2:21-26.

14. Crona DJ, Keisler MD, Walko CM. Regorafenib: a novel multitargeted tyrosine kinase inhibitor for colorectal cancer and gastrointestinal stromal tumors. Ann Pharmacother. 2013;47(12):1685-1696.

15. Grothey A, Van Cutsem E, Sobrero A, et al. Regorafenib monotherapy for previously treated metastatic colorectal cancer (CORRECT): an international, multicentre, randomised, placebo-controlled, phase 3 trial. Lancet. 2013;381(9863):303-312.

16. De Wit M, Boers-Doets CB, Saettini A, et al. Prevention and management of adverse events related to regorafenib. Support Care Cancer. 2014;22(3):837-846.

17. Schultheis B, Folprecht G, Kuhlmann J, et al. Regorafenib in combination with FOLFOX or FOLFIRI as first- or second-line treatment of colorectal cancer: results of a multicenter, phase Ib study. Ann Oncol. 2013;24(6):1560-1567.
OncoTargets and Therapy

\section{Publish your work in this journal}

OncoTargets and Therapy is an international, peer-reviewed, open access journal focusing on the pathological basis of all cancers, potential targets for therapy and treatment protocols employed to improve the management of cancer patients. The journal also focuses on the impact of management programs and new therapeutic agents and protocols on

\section{Dovepress}

patient perspectives such as quality of life, adherence and satisfaction The manuscript management system is completely online and includes a very quick and fair peer-review system, which is all easy to use. Visit http://www.dovepress.com/testimonials.php to read real quotes from published authors. 